[20] Narin, R., Narin, M. A., Nazik, H. et. al. (2015). The importance of size of cervical ectopy to predict postcoital bleeding: is there any cut-off value? Clinical and Experimental Obstetrics and Gynecology, $42(2), 195-198$.

[21] Singh, V., Parashari, A., Satyanarayana, L., Sodhani, P., Gupta, M. M., Sehgal, A. (1999). Biological behavior and etiology of inflammatory cervical smears. Diagnostic Cytopathology, 20 (4), 199-202. doi: 10.1002/(sici)1097-0339(199904)20:4<199::aid-dc3>3.0.co;2-2

[22] Sanchez, A., Rivera, A., Castillo, F., Ortiz, S. (2012). Cervical erosion as result of infectious vaginitis. European Journal of Experimental Biology, 2 (5), 1659-1663.

[23] Trejo, J., Hernandez, B., Carrasco, J. et. al. (2003). Guia clinica para el diagnostico, tratamiento y prevencion de cervicovaginitis por bacterias, Trichomonas y Candida. Revista Medica del Instituto Mexicano del Seguro Social, 41, 71-76.

\title{
PROGNOSTIC VALUE OF IMMUNOHISTOCHEMICAL MARKERS IN PATIENTS WITH DIFFUSE LARGE B-CELL LYMPHOMA
}

\author{
Arina Martynchyk \\ Scientific Research Department of Chemotherapy of Hemoblastoses and Adjuvant Treatment Methods \\ National Cancer Institute \\ 33/43 Lomonosova str., Kyiv, Ukraine, 03022 \\ martynchyk.arina@gmail.com \\ Vitaliy Gurianov \\ Department of Medical Physics \\ Bogomolets National Medical University \\ 13 Shevchencko ave., Kyiv, Ukraine, 01601 \\ i_@ukr.net \\ Iryna Kriachok \\ Scientific Research Department of Chemotherapy of Hemoblastoses and Adjuvant Treatment Methods \\ National Cancer Institute \\ 33/43 Lomonosova str., Kyiv, Ukraine, 03022 \\ irina.kryachok@unci.org.ua
}

\begin{abstract}
Diffuse large B-cell lymphoma (DLBCL) is a potentially curable disease, but standard treatment is not effective enough for all patients. That is why so important to identify high risk patients who need more aggressive therapy at the time of diagnosis. Nowadays prognosis for patients with DLBCL is based on International prognostic index (IPI). However, this index consists of only clinical parameters and does not include the biological characteristics of the tumour. Immunohistochemistry (IHC) markers could also play a prognostic role. There are some publications regarding predictive and prognostic role of expression of Bcl-2, Bcl-6, MUM1, $\mathrm{CD} 10$ and CD30, but their results are controversial. The aim of our study was to analyze prognostic role of these markers, to compare survival of patients with positive and negative expression of these markers and to build a prognostic model which include biological parameters for identifying high risk patients. There were statistically significant differences in EFS between the group of patients with negative and positive expression of CD10 (51.5\% versus $72.5 \%, \mathrm{p}=0.01)$ and in OS between the group of patients with negative and positive expression of Bcl-6 (61.1\% versus 79.6\%, $\mathrm{p}=0.03)$. Six-factors nonlinear neural network prediction model (MLP_6) was built. The sensitivity of the model is $63.2 \%$ (95 \% CI $49.3 \%-75.6 \%)$, specificity $-85.2 \%$ (95\% CI $79.1 \%-90.1 \%)$. Prognostic factors include negative IHC expression of Bcl-6, CD10, non-GCB molecular subtype (according to algorithm Hans), gender (male), advanced Ann-Arbor stages, $>2$ extranodal involvement. Our nonlinear neural network prediction model could improve prognostic role of IPI by adding of biological tumour characteristics (IHC expression of CD10, Bcl-6, molecular subtype by IHC algorithm).

Keywords: diffuse large B-cell lymphoma, prognosis, predictive model, biological characteristics.
\end{abstract}




\section{Introduction}

Diffuse large B-cell lymphoma (DLBCL) is a potentially curable disease, but unfortunately approximately $40 \%$ of patients are refractory to the primary therapy or develop relapse after first line therapy $[1,2]$.

Standard of treatment is not effective enough for this group of patients. That is why so important to identify high risk patients who need more aggressive therapy at the time of diagnosis. On the other hand there are a lot of new drugs recently and searching for prognostic markers is extremely important for risk stratification of patients in order to individualize treatment.

Nowadays prognosis for patients with DLBCL is based on International prognostic index (IPI) [3]. IPI was described in1993 based on the analysis of 3273 patients with aggressive non-Hodgkin lymphoma in1 6 Oncologic centers, received CHOP-like treatment. Based on such negative prognostic factors as age more than 60 years old, elevated lactate dehydrohynase, status ECOG $>1$, III-IV stages, extranodal sites of involvement $>1$, patients were divided into four risk groups: low ( $0-1$ factor), low-intermediate ( 2 factors), high-intermediate (3 factors) and high risk (4-5 factors) with 5-year overall survival (OS) $73 \%, 51 \%, 43 \%$ i $26 \%$ accordingly [3]. However, despite its wide spread use in clinical practice and predictive value, this index consists of only clinical parameters and does not include the biological characteristics of the tumour. Immunohistochemistry (IHC) markers which are used for diagnosis of lymphoma and play important role in its pathogenesis could also play a prognostic role. There are some publications regarding predictive and prognostic role of expression of Bcl-2, Bcl-6, MUM1, CD10 and CD30, but their results are controversial.

Positive expression of MUM1 is associated with inferior outcome according to the several study results $[4,5]$. And some study results demonstrated positive prognosis in case of positive expression of this marker [6, 7].

According to the results of Berglund and Sjöetal positive expression of CD10 is a positive prognostic factor $[8,9]$ and have no prognostic impact according other results [10].

Negative prognostic influence of Bcl-2 was demonstrated in several studies [8, 11]. And according to other results there is no prognostic value of this marker [12, 13].Negative Bcl-6 expression is associated with inferior survival according Horn and his colleagues [14, 15] and there is no prognostic value of this marker according others results [16].

\section{Aim of research}

The aim of the study was to identify the prognostic role of immunohistochemical markers and to find prognostic model for detecting patients with survival less than 12 months.

\section{Materials and methods}

In the retrospective analysis were included 349 patients with newly diagnosed DLBCL who were treated at the National Cancer Institute (Kyiv, Ukraine) from December, 2014 to December, 2017. IHC expression of Bcl-2, Bcl-6, MUM1, C-MYC, CD10 or CD30 in these patients were compared.

IPI factors and survival between groups of patients with positive and negative expression of IHC markers were compared. For identifying of molecular subtype (germinal center (GCB) DLBCL or non-germinal center DLBCL (non-GCB)) immunohistochemical Hans algorithm was used [17].

Group comparisons were performed by means of $\chi^{2}$ and Student's t tests. For time to event analyses we used EZR v. 1.35 (Saitama Medical Center, Jichi Medical University, Saitama, Japan, 2017), applying Kaplan-Meier survival estimates with the end-point of overall survival, defined as the time from initial diagnosis to death from any cause [18]. P values less than 0.05 were considered statistically significant.

For the analysis of the risk of death in the first 12 months after diagnosis and construction of the system for predicting this risk, the method of analysis of neural network models [19] was used. To select a minimum set of parameters related to the risk of early death, the method of genetic algorithm for selecting variables [19] was used.

In order to assess the adequacy of the forecasting model, the method of constructing and analyzing the operational characteristics curves of the models (ROC curves) was used, the estimation 
of the quality of the prediction was made by assessing of area under the operational characteristics curve (AUC), sensitivity, specificity, predictability of the positive result, and the prediction of the negative outcome, the corresponding $95 \%$ of the indicators were calculated [20].

\section{Result}

There were $162(46.4 \%)$ men and $187(53.6 \%)$ women. Age of patients was from 17 to 86 years, median age $-54.0 \pm 1.1$ years of old (95\% CI 50-56). Early stages (I-II) were registered in $182(52.1 \%)$ of patients, advanced stages (III-IV) - in $167(47.9 \%)$. There were B-symptoms in $127(36.4 \%)$ of patients. There was low and low-intermediate IPI in $238(68.2 \%)$ of patients and $111(31.8 \%)$ high and high-intermediate risk patients. There were 132 (37.8\%) patients older than 60 years old, 153 (43.8 \%) patients with elevated LDH, 167 (47.9\%) patients with advanced stages, $89(25.5 \%)$ patients with ECOG $>2$ and $99(28.4 \%)$ patients with more than two extranodal sites of involvement. Rituximab-based chemotherapy received 186 of patients $(53.3 \%)$, patients received 1-8 courses of chemotherapy (6 \pm 0.1 courses, $95 \%$ CI 5.0-7.0). Radiation therapy received 116 of patients (33.2\%). Median follow up was 12.7 months.

Positive expression of Bcl-2, Bcl-6, MUM1, C-MYC, CD10 or CD30 was registered in $79.4 \%, 56.8 \%, 63.7 \%, 28 \%, 38.2 \%$, and $23.5 \%$ accordingly (Fig. 1).

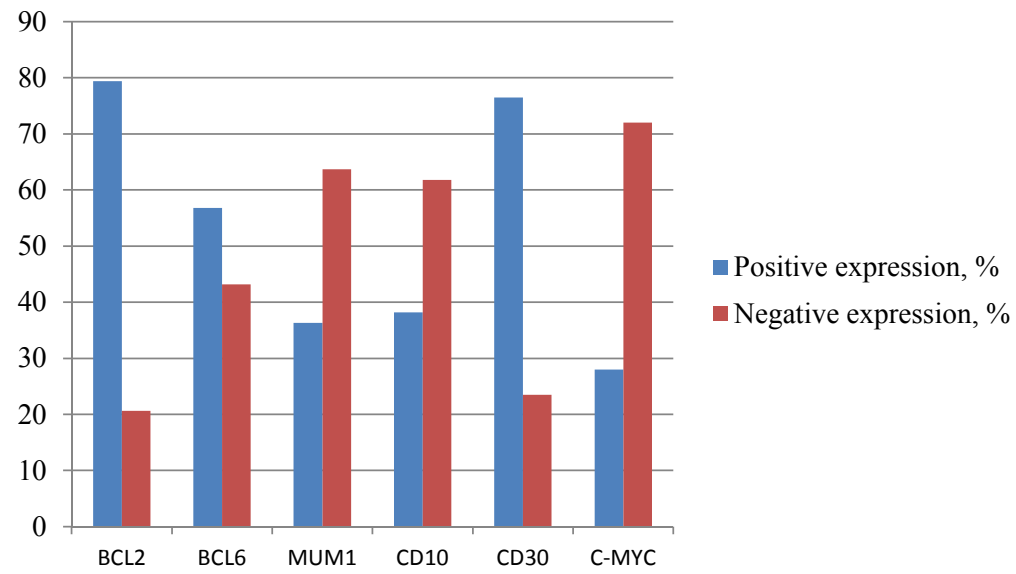

Fig. 1. Incidence of positive (blue color) and negative (red color) expression of IHC markers

In the Table 1 IPI factors distribution depending on positive or negative expression of IHC markers is demonstrated.

Table 1

IPI risk factors in the group with positive and negative expression of IHC markers

\begin{tabular}{|c|c|c|c|c|c|c|}
\hline$\%$ IHC & Age $>60$ & Stage III-IV & $\begin{array}{c}>2 \text { extranodal } \\
\text { sites }\end{array}$ & $\mathbf{L D N}>\mathbf{N}$ & ECOG $>2$ & IPI 3-5 \\
\hline 1 & 2 & 3 & 4 & 5 & 6 & 7 \\
\hline Bcl-2+ & 42 & 53.5 & 32 & 55.3 & 28 & 38 \\
\hline Bcl-2- & 28.8 & 35 & 23 & 63.6 & 23 & 21 \\
\hline $\mathrm{p}$ & 0.12 & 0.08 & 0,27 & 0.41 & 0.69 & 0.04 \\
\hline Bcl-6+ & 33.3 & 45.8 & 31,9 & 59 & 25.4 & 34.2 \\
\hline Bcl-6- & 39.5 & 56 & 32,9 & 63,4 & 31.8 & 35.1 \\
\hline $\mathrm{p}$ & 0.43 & 0,18 & 0.99 & 0.68 & 0.38 & 0.99 \\
\hline MUM1+ & 41.5 & 44.5 & 24.4 & 55.8 & 24.4 & 32.3 \\
\hline MUM1- & 35.1 & 44.5 & 31.1 & 52.2 & 22.9 & 28.3 \\
\hline
\end{tabular}


Continuation of Table 1

\begin{tabular}{ccccccc}
\hline 1 & 2 & 3 & 4 & 5 & 6 & 7 \\
\hline $\mathrm{p}$ & 0.45 & 0.7 & 0.67 & 0.76 & 0.95 & 1.0 \\
$\mathrm{CD} 10+$ & 30.9 & 38 & 27.4 & 51.3 & 22.6 & 27.4 \\
$\mathrm{CD} 10-$ & 42.6 & 51.4 & 30.4 & 55 & 25 & 33.8 \\
$\mathrm{p}$ & 0.11 & 0.053 & 0.75 & 0.74 & 0.82 & 0.39 \\
$\mathrm{CD} 30+$ & 25.9 & 44.4 & 33.3 & 54.5 & 22.2 & 18.5 \\
$\mathrm{CD} 30-$ & 38.6 & 54.4 & 26.4 & 50 & 27.7 & 31.8 \\
$\mathrm{p}$ & 0.32 & 0.48 & 0.97 & 0.89 & 0.91 & 0.27 \\
$\mathrm{C}-\mathrm{MYC}+$ & 37.1 & 57.1 & 37.1 & 51.5 & 40 & 42.8 \\
$\mathrm{C}-\mathrm{MYC}-$ & 44.4 & 54.4 & 38.2 & 72.7 & 32.5 & 50
\end{tabular}

There were no difference in age $>60$ years old, advanced stages, elevated LDH, more than two extranodal involvements, ECOG $>2$ between patients with positive and negative expression of Bcl-2, Bcl-6, CD10, CD30, MUM1 та C-MYC. There was similar distribution of high risk patient between with positive and negative expression of Bcl-6, CD10, CD30, MUM1 та C-MYC. There were more high risk patients according IPI in the group of patients with positive Bcl-2 expression $(\mathrm{p}=0.04)$.

Two-year overall survival (OS) and event-free survival (EFS) were analyzed and compared between group of patients with positive and negative expression of IHC markers (Table 2).

Table 2

Two-year overall and event-free survival in patients with positive and negative expression of IHC markers

\begin{tabular}{|c|c|c|c|c|}
\hline IHC, \% & EFS, \% & $95 \%$ CI & OS, \% & $95 \%$ CI \\
\hline Bcl-2+ & $65.5 \pm 7.35$ & $49.1-77.8$ & $73.23 .8 \pm$ & $64.8-79.8$ \\
\hline Bcl-2- & $61.44 .1 \pm$ & $52.9-68.9$ & $76.36 .7 \pm$ & $59.9-86.6$ \\
\hline $\mathrm{p}$ & 0.6 & & \multicolumn{2}{|c|}{0.8} \\
\hline Bcl-6+ & $52.96 .36 \pm$ & $39.8-64.4$ & $79.64 .3 \pm$ & $69.5-86.6$ \\
\hline Bcl-6- & $63.25 .17 \pm$ & $52.2-72.4$ & $61.16 .3 \pm$ & $47.6-72.1$ \\
\hline $\mathrm{p}$ & 0.2 & & \multicolumn{2}{|c|}{0.03} \\
\hline MUM1+ & $63.04 .86 \pm$ & $52.7-71.7$ & $77.04 .9 \pm$ & $65.5-85.1$ \\
\hline MUM1- & $57.07 .21 \pm$ & $41.8-69.7$ & $66.76 .8 \pm$ & $51.5-78.1$ \\
\hline $\mathrm{p}$ & 0.7 & & \multicolumn{2}{|c|}{0.1} \\
\hline CD10+ & $72.55 .58 \pm$ & $59.8-81.7$ & $77.05 .8 \pm$ & $62.9-86.3$ \\
\hline CD10- & $51.55 .38 \pm$ & $42.7-63.0$ & $68.35 \pm$ & $57.1-77.1$ \\
\hline $\mathrm{p}$ & 0.01 & & \multicolumn{2}{|c|}{0.2} \\
\hline CD30+ & $53.111 .1 \pm$ & $29.9-71.8$ & $65.410 .9 \pm$ & $39.9-82.2$ \\
\hline CD30- & $54.66 .6 \pm$ & $40.8-66.4$ & $68.66 .4 \pm$ & $54.1-79.3$ \\
\hline $\mathrm{p}$ & 0.9 & & 0.805 & \\
\hline MYC+ & $47.912 .85 \pm$ & $22.2-69.7$ & $67.514 .3 \pm$ & $32.2-87.3$ \\
\hline MYC- & $59.46 .03 \pm$ & $46.6-70.0$ & $69.06 .15 \pm$ & $55.2-79.3$ \\
\hline $\mathrm{p}$ & 0.9 & & 0.5 & \\
\hline
\end{tabular}


In the group of patients with negative expression of CD10 there EFS was worse comparing to the group of patients with positive expression of CD10 (51.5\% versus $72.5 \%, \mathrm{p}=0.01)$. In the group of patients with negative expression of Bcl-6 there was worse OS comparing to the group of patients with positive expression of Bcl-6 (61.1\% versus $79.6 \%, \mathrm{p}=0.03)$ (Fig. 2).

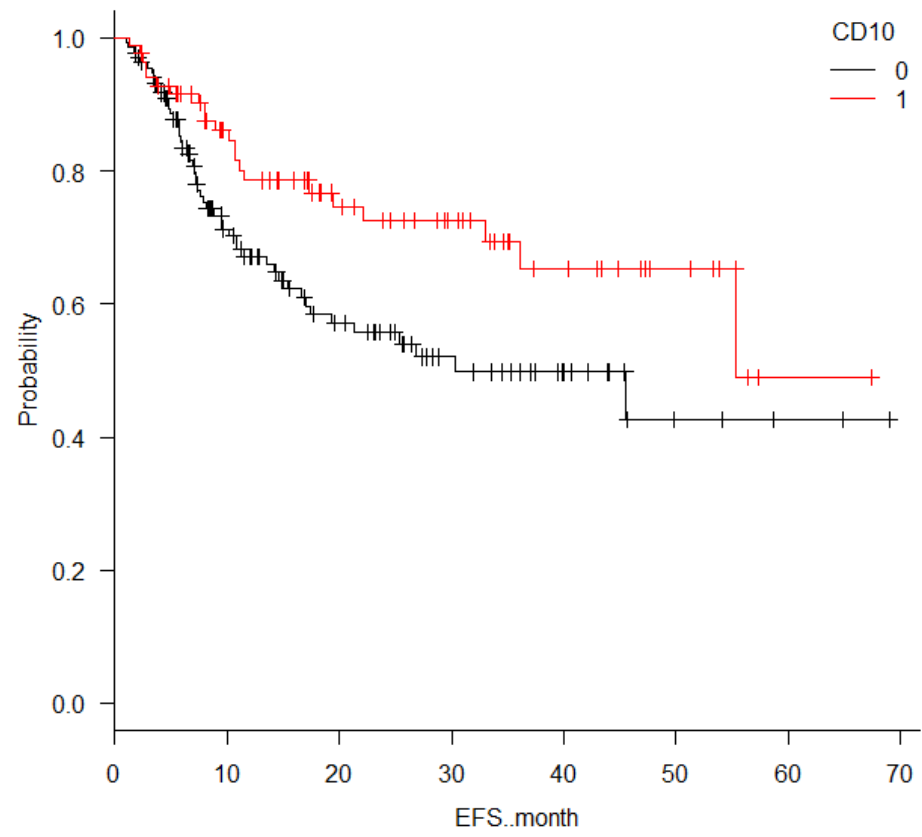

$a$

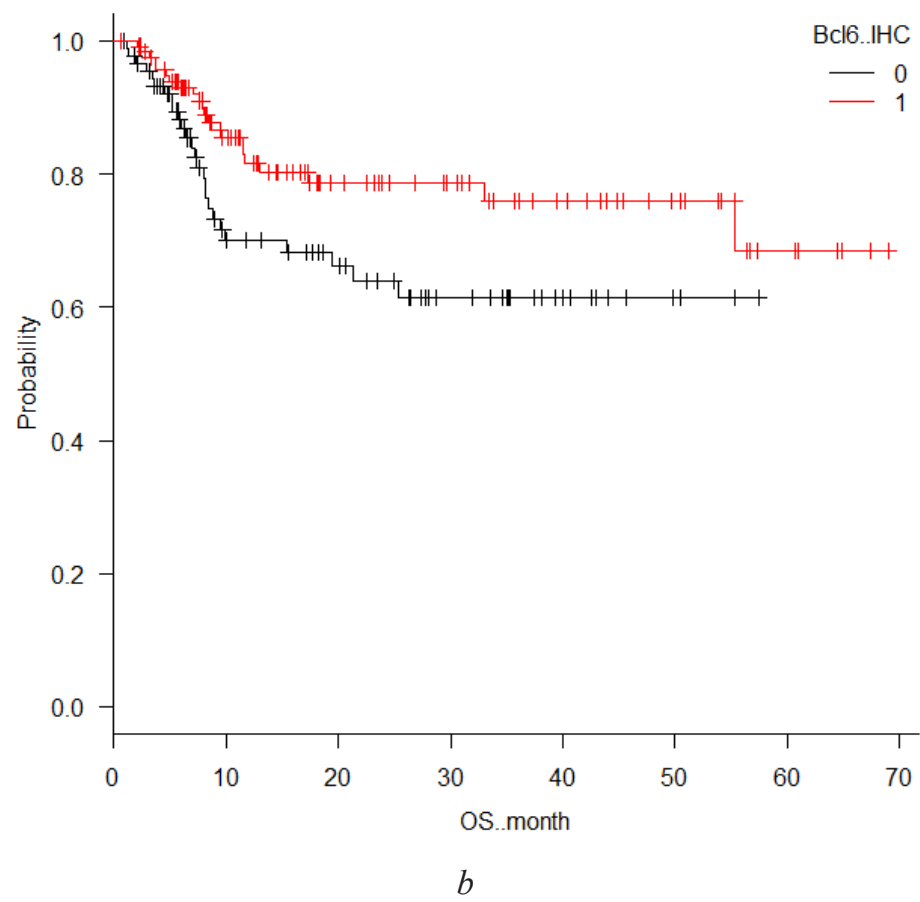

Fig. 2. Survival of DLBCL patients depending on IHC markers expression:

$a-$ EFS of patients with positive (1, red line) and negative ( 0 , black line) expression of CD10;

$b-$ OS of patients with positive (1, red line) and negative ( 0 , black line) expression of Bcl- 6

An analysis of the association of treatment results of patients with DLBCL with different factor characteristics (27 clinical characteristics of patients, including IPI factors, IHC expression 
of Bcl-2, MUM1, Bcl-6, CD10, CD30, C-MYC etc.) was performed. As a starting point, the likelihood of survival for more than 12 months was chosen. Outcome variable $\mathrm{Y}=0$ for patients with OS $>12$ months (176 patients), $\mathrm{Y}=1$ for patients with OS 12 months or less (57 patients).

The 27-factor linear neural network model for prediction survival less than 12 month was built with $79.3 \%$ (95\% IU 58.5-83.0\%) of sensitivity and $80.1 \%$ (95\% IU $73.4 \%-85.7 \%$ ) of specificity.

As a result of selection of the minimum number of the most significant factors the method of genetic selection algorithm was used [1]. There were six characteristics for prognosing survival $<12$ months: negative IHC expression of Bcl-6 (X1), CD10 (X2), non-GCB molecular subtype (according to algorithm Hans) (X3), gender (male) (X4), advanced Ann-Arbor stages (X5), >2extranodal involvement (X6). Based on this characteristics 6-factor linear prediction model (Lin_6) with $86.0 \%$ (95\% CI $74.2 \%-93.7 \%$ ) of sensitivity and $62.5 \%$ (95 \% CI $54.9 \%-69.7 \%$ ) of specificity was built.

In order to find out the possible nonlinear relationships of the distinguished factor characteristics and the risk of low survival, in the third stage of the analysis, a six-factors nonlinear neural network prediction model (such as a multi-layered perceptron with one hidden layer) (MLP_6) with the same parameters was built. The sensitivity of the model is $63.2 \%$ (95\% CI $49.3 \%-\overline{7}$. $5.6 \%$ ), specificity $-85.2 \%$ (95\% CI $79.1 \%-90.1 \%)$. Fig. 3 shows the architecture of this model.

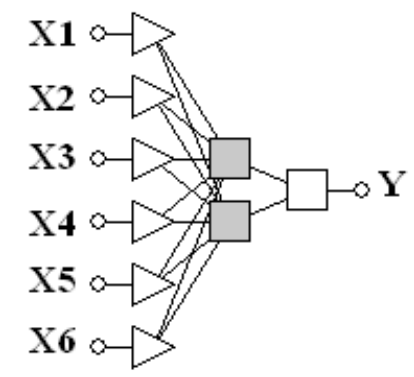

Fig. 3. Architecture of six-factor nonlinear neural network prediction model for prognosis of low survival. White triangles are the neurons of the input layer, the gray squares - the hidden layer neurons, the white square - the neuron of the initial layer of the network

The method of comparison of curves of operational characteristics of models was used for comparison of prognosis qualities of three constructed predicting models: Lin_27, Lin_6 and MLP_6 (Fig. 4).

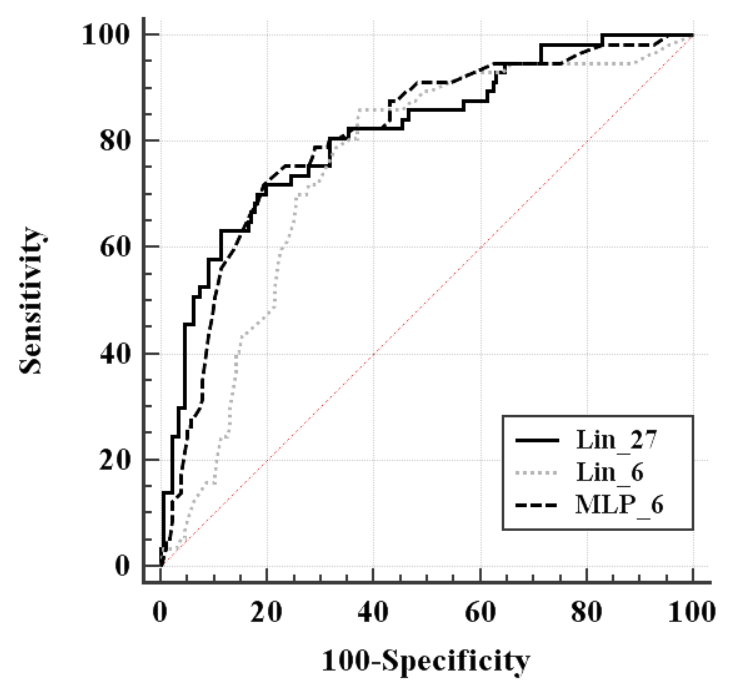

Fig. 4. Curves of operational characteristics of prognosis qualities of three constructed predicting models 
According to this analysis of ROC-curves there were no differences between Lin_27 and MLP_6.

To choose the optimal threshold, the decision was made based on minimizing the prediction error (minimizing the error of false negative + false positive results). At the same time, the same value of errors was chosen. When conducting an optimization, the threshold value Ycrit $=0.527$, while $\mathrm{Y} \leq$ Ycrit in the model predicts the risk of low survival, otherwise the prognosis is favorable.

Group of patients with positive and negative expression of IHC markers Bcl-2, Bcl-6, CD10, CD30, MUM1 та C-MYC were comparable in such characteristics as old age, advanced stages, elevated $\mathrm{LDH}$, more than two extranodal involvements, $\mathrm{ECOG}>2$. There were more high risk patients according IPI in the group of patients with positive Bcl-2 expression ( $p=0.04)$.

There were statistically significant difference in EFS between the group of patients with negative and positive expression of $\operatorname{CD} 10(51.5 \%$ versus $72.5 \%, \mathrm{p}=0.01)$ and in OS between the group of patients with negative and positive expression of Bcl-6 (61.1\% versus $79.6 \%, \mathrm{p}=0.03)$.

Six-factors nonlinear neural network prediction model (MLP_6) was built. The sensitivity of the model is $63.2 \%(95 \%$ CI $49.3 \%-75.6 \%)$, specificity - $85.2 \%$ (95\% CI $79.1 \%-$ $90.1 \%$ ). Prognostic factors include negative IHC expression of Bcl-6, CD10, non-GCB molecular subtype (according to algorithm Hans), gender (male), advanced Ann-Arbor stages, >2 extranodal involvement.

\section{Discussion}

Despite more numbers of high risk patients according IPI were in the group of patients with positive Bcl-2 expression, expression of this IHC marker did not have any influence on survival and did not show significant value during multivariable analysis. The results of study by Wilson W. H. and colleagues also demonstrated the absence of prognostic role of Bcl-2 expression.

Our analysis suggests association between negative IHC expression of CD10 and Bcl-6 and inferior outcome in patients with DLBCL.According to the Hans' et al. and Berglund's et al. data, positive expression of $\mathrm{CD} 10$ and Bcl6 also are associated with better survival.

This suggestion was also confirm in multivariable analysis where six independent factors of poor survival were identified. Apart from 2 IPI factors (age, extranodal involvements) 1 new clinical factor (gender) and 3 biological tumor characteristics were identified. This combination of three new biological factors in our prognostic model was not described in the literature before and is valuable addition to the IPI for identifying poor prognosis patients with DLBCL.

\section{Conclusion}

1. IPI is a standard, but not perfect predictor of prognosis in patients with DLBCL and it includes only clinical patients' characteristics.

2. Our nonlinear neural network prediction model could improve prognostic role of IPI by adding of biological tumor characteristics (IHC expression of CD10, Bcl-6, molecular subtype by IHC algorithm).

3. This prognostic model needs further confirmation in larger studies.

\section{References}

[1] Swerdlow, S. H., Campo, E., Pileri, S. A., Harris, N. L., Stein, H., Siebert, R. et. al. (2016). The 2016 revision of the World Health Organization classification of lymphoid neoplasms. Blood, 127 (20), 2375-2390. doi: 10.1182/blood-2016-01-643569

[2] Sehn, L. H. (2014). Diffuse large B-cell lymphoma: one treatment no longer fits all. Oncology, 28 (4), 334-336.

[3] A Predictive Model for Aggressive Non-Hodgkin's Lymphoma. The International Non-Hodgkin's Lymphoma Prognostic Factors Project (1993). New England Journal of Medicine, 329 (14), 987-994. doi: 10.1056/nejm199309303291402

[4] Van Imhoff, G. W., Boerma, E.-J. G., van der Holt, B., Schuuring, E., Verdonck, L. F., Kluin-Nelemans, H. C., Kluin, P. M. (2006). Prognostic Impact of Germinal Center-Associated Proteins and 
Chromosomal Breakpoints in Poor-Risk Diffuse Large B-Cell Lymphoma. Journal of Clinical Oncology, 24 (25), 4135-4142. doi: 10.1200/jco.2006.05.5897

[5] Amen, F., Horncastle, D., Elderfield, K., Banham, A. H., Bower, M., Macdonald, D. et. al. (2007). Absence of cyclin-D2 and Bcl-2 expression within the germinal centre type of diffuse large B-cell lymphoma identifies a very good prognostic subgroup of patients. Histopathology, 51 (1), 70-79. doi: 10.1111/j.13652559.2007.02721.x

[6] Hallack Neto, A. E., Dulley, F. L., Coelho Siqueira, S. A., Pracchia, L. F., Belesso, M., Saboya, R. et. al. (2008). Prognostic impact of diffuse large B-cell lymphoma subgroups in patients undergoing autologous SCT. Bone Marrow Transplantation, 43 (4), 323-325. doi: 10.1038/bmt.2008.330

[7] Kryachok, I., Martynchyk, A. V., Filonenko, K., Grabovoy, A. N., Antoniuk, S. A., Tytorenko, I. et. al. (2016). The predictive value of immunohistochemical expression of Bcl-2, Bcl-6, MUM1, CD10 and CD30 in patients with diffuse large cell lymphoma. Annals of Oncology, 27, 923. doi: 10.1093/annonc/ mdw375.18

[8] Berglund, M., Thunberg, U., Amini, R. M. et. al. (2005). Evaluation of immunophenotype in diffuse large B-cell lymphoma and its impact on prognosis. Modern Pathology, 18, 1113-1120.

[9] Sjo, L. D., Poulsen, C. B., Hansen, M., Moller, M. B., Ralfkiaer, E. (2007). Profiling of diffuse large B-cell lymphoma by immunohistochemistry: identification of prognostic subgroups. European Journal of Haematology, 79 (6), 501-507. doi: 10.1111/j.1600-0609.2007.00976.x

[10] Fabiani, B., Delmer, A., Lepage, E., Guettier, C., Petrella, T. et. al. (2004). CD10 expression in diffuse large B-cell lymphomas does not influence survival. Virchows Archiv, 445 (6), 545-551. doi: 10.1007/ s00428-004-1129-7

[11] Barrans, S. L., O’Connor, S. J. M., Evans, P. A. S., Davies, F. E., Owen, R. G., Haynes, A. P. et. al. (2002). Rearrangement of the BCL6 locus at 3q27 is an independent poor prognostic factor in nodal diffuse large B-cell lymphoma. British Journal of Haematology, 117 (2), 322-332. doi: 10.1046/j.13652141.2002.03435.x

[12] Wilson, W. H., Dunleavy, K., Pittaluga, S., Hegde, U., Grant, N., Steinberg, S. M. et. al. (2008). Phase II Study of Dose-Adjusted EPOCH and Rituximab in Untreated Diffuse Large B-Cell Lymphoma With Analysis of Germinal Center and Post-Germinal Center Biomarkers. Journal of Clinical Oncology, 26 (16), 2717-2724. doi: 10.1200/jco.2007.13.1391

[13] Muris, J., Meijer, C., Vos, W., van Krieken, J., Jiwa, N., Ossenkoppele, G., Oudejans, J. (2006). Immunohistochemical profiling based on Bcl-2, CD10 and MUM1 expression improves risk stratification in patients with primary nodal diffuse large B cell lymphoma. The Journal of Pathology, 208 (5), 714-723. doi: $10.1002 /$ path.1924

[14] Horn, H., Ziepert, M., Becher, C., Barth, T. F. E., Bernd, H.-W. et. al. (2013). MYC status in concert with BCL2 and BCL6 expression predicts outcome in diffuse large B-cell lymphoma. Blood, 121 (12), 2253-2263. doi: 10.1182/blood-2012-06-435842

[15] Colomo, L., Lopez-Guillermo, A., Perales, M. et. al. (2003). Clinical impact of the differentiation profile assessed by immunophenotyping in patients with diffuse large B-cell lymphoma. Blood, 101 (1), 78-84. doi: 10.1182/blood-2002-04-1286

[16] Linderoth, J., Jerkeman, M., Cavallin-Stahl, E. et. al. (2003) Immunohistochemical expression of CD23 and CD40 may identify prognostically favorable subgroups of diffuse large B-cell lymphoma: a Nordic Lymphoma Group Study. Clinical Cancer Research, 9 (2), 722-728.

[17] Hans, C. P., Weisenburger, D. D., Greiner, T. C. et. al. (2004). Confirmation of the molecular classification of diffuse large B-cell lymphoma by immunohistochemistry using a tissue microarray. Blood, 103 (1), 275-282. doi: 10.1182/blood-2003-05-1545

[18] Kanda, Y. (2012). Investigation of the freely available easy-to-use software "EZR" for medical statistics. Bone Marrow Transplantation, 48 (3), 452-458. doi: 10.1038/bmt.2012.244

[19] Liah, Y., Gurianov, G. (2012). Mathematical modeling in solving classification problems in biomedicine. Ukrainskyi zhurnal telemedycyny ta medychnoi telematyky, 10 (2), 69-76.

[20] Petrie, A., Sabin, C. (2009). Medical Statistics at a Glance. Wiley-Blackwell, 180. 